\title{
Repercusiones en la Cavidad Oral Causadas por la Infección con COVID-19
}

\author{
Repercussions on the Oral Cavity Caused by COVID-19 Infection
}

\author{
Britto E. Falcón-Guerrero \& Guido S. Falcón-Pasapera²
}

FALCÓN-GUERRERO, B. E. \& FALCÓN-PASAPERA, G. S. Repercusiones en la cavidad oral causadas por la infección con COVID-19. Int. J. Odontostomat., 15(1):23-26, 2021.

RESUMEN : Ante esta situación de la pandemia por el COVID-19, los odontólogos tienen un papel importante para detectar y apoyar en el diagnóstico temprano, dado que se están reportando efectos que esta infección ocasiona en la cavidad oral y que se relacionan con el inicio de este proceso infeccioso. Estos efectos se pueden relacionar con la presencia del virus SARS-CoV-2 o con las formas de los tratamientos que se le brinda al paciente, que van en desmedro de la salud oral de los pacientes. El objetivo de esta revisión es informar sobre las repercusiones que se pueden dar en la cavidad oral por la infección con COVID-19.

PALABRAS CLAVE: COVID-19, Servicios de Salud Dental, SARS-CoV-2; control de infecciones, coronavirus, saliva, odontología.

\section{INTRODUCCIÓN}

El mundo se encuentra en una situación de constante riesgo debido a la aparición del virus SARS-CoV-2 que genera la enfermedad del COVID19 , el cual se ha diseminado de manera exponencial en todos los países del orbe provocando desafíos y cambios significativos en la forma de prestar los servicios de salud, incluida la actividad de odontología. Pero se puede aprender de esta situación para replantear la función del odontólogo dentro del equipo de atención para una atención más adaptable y centrado al paciente, lo que motiva a redefinir y prepararnos mejor para el futuro, cambiando nuestra mentalidad y ampliando nuestros conocimientos sobre las repercusiones de esta enfermedad. (Falcón-Guerrero \& Falcón-Pasapera, 2020; Dziedzic, 2020).

Durante la práctica dental el riesgo de exponerse a SARS-CoV-2 es muy alto debido a la cercanía con la cavidad oral y la nariz del paciente, la exposición a saliva, sangre y otros fluidos corporales y al manejo de instrumental rotatorio que generan aerosoles infecciosos que exponen al odontó- logo a infectarse, ya que este virus se une inicialmente a la enzima convertidora de angiotensina II (ACE2) que se encuentra en el epitelio de la mucosa oral, para después recién colonizar los tractos respiratorios (Peng et al., 2020).

En este contexto, se han reportado ciertas manifestaciones clínicas a nivel de la cavidad oral, aunque estas aún pueden ser escasas debido a que los odontólogos han sido separados de su actividad clínica por los riesgos de contaminación, lo que puede enmascarar la real repercusión del COVID19 en la cavidad oral (Cornejo \& Espinoza, 2020).

Ante esta problemática, un factor importante a tener en cuenta para poder lograr manejar esta enfermedad de forma temprana, es que los odontólogos tengan los conocimientos necesarios sobre las repercusiones que se presentan en la cavidad oral a consecuencia del virus SARS-CoV-2. El objetivo de esta revisión es informar sobre las repercusiones que se pueden dar en la cavidad oral por la infección con COVID-19.

\footnotetext{
${ }^{1}$ Facultad de odontología, Universidad Latinoamericana CIMA. Tacna, Perú.

${ }^{2}$ Facultad de odontología, Universidad Peruana Cayetano Heredia. Lima, Perú.
} 


\section{LA MUCOSA ORAL COMO RECEPTOR DEL SARS- CoV-2}

Se ha reportado que el receptor de la ACE2 actúa como vía principal para la entrada del SARSCoV-2 a la célula huésped causando la infección final. Este ACE2 está presente en el tejido oral y gingival, convirtiendo a sus células en más susceptibles a la infección, lo que explica el mecanismo básico de que la cavidad oral tiene un riesgo potencialmente alto para la infección con SARS-CoV-2. (Xu et al., 2020a,b; To et al., 2020). En este sentido las células epiteliales de las glándulas salivales y de la lengua actúan como anfitriones de SARS-CoV2 gracias a que poseen una alta expresión de ACE2. (Xu et al., 2020a,b; Liu et al., 2011) Entendiéndose que la cavidad oral se infecta más fácilmente ya que actúa como anfitriona para iniciar la invasión de este virus (Srinivasan et al., 2020).

Por otra parte, se conoce que los virus usan el ACE2 para su entrada y para que se establezcan en el pulmón dependen de la proteasa transmembrana de serina 2 asociada a la superficie del huésped para recién infectar las células epiteliales cuboides de tipo 2 (célula AT2, que es el objetivo principal del SARS-CoV-2) en el pulmón (Qi et al., 2020).

También se ha demostrado la existencia del SARS-CoV-2 en la saliva de pacientes infectados, considerándose que la cavidad oral tiene un rol importante para la trasmisión de este virus dentro del ambiente odontológico entre persona y persona. Este hecho también se puede considerar para hacer un diagnóstico no invasivo, gracias a la medición de ACE-2 en la saliva que podría ser un marcador de la infección por COVID-19 durante su fase temprana (Srinivasan et al.; Sabino-Silva et al., 2020).

Sabino-Silva et al., manifiestan que pueden haber tres vías diferentes para que el SARS-CoV-2 se presente en la saliva: (1) Desde el tracto respiratorio inferior y superior que se comunican a la cavidad oral junto con las gotitas de líquido frecuentemente intercambiadas por estos órganos. (2) La presencia del SARS-CoV-2 en la sangre, que puede acceder a la cavidad oral a través del fluido crevicular. (3) Por infección de las glándulas salivales mayor y menor, con la posterior liberación de partículas en la saliva a través de los conductos salivales; donde las glándulas salivales pueden ser una fuente fundamental de este virus.

\section{REPERCUSIONES EN LA CAVIDAD ORAL}

Los posibles síntomas relacionados con la cavidad oral incluyen hipogeusia, xerostomía y alteraciones quimiosensoriales, destacándose que pacientes positivos a COVID-19 presentan diferentes grados de gravedad y aparición de alteraciones del gusto (Soares et al., 2020; Harikrishnan et al., 2020) La anosmia y la ageusia son las manifestaciones más comunes de SARS-COV-2 en el sistema nervioso periférico, y se presentan como signó inicial de esta enfermedad, por lo tanto se sugiere que las personas con disfunciones gustativas pueden ser posibles portadores y deben aislarse de los demás. Se ha propuesto como mecanismos asociados que los coronavirus son neurotrópicos y neuroinvasores, lo que puede conducir a una alteración del gusto a través de los nervios craneales; también se sugiere que la presencia de la ACE2 es más alta en el epitelio de la lengua que en los tejidos orales y gingivales, por lo que la afectación del epitelio de la lengua puede contribuir a la alteración del gusto; aunque la causa exacta aun un está determinada (Harikrishnan et al.; Niazkar et al., 2020; Lee \& Lee., 2020).

Por lo tanto, se ha asociado la pérdida del sentido del gusto en hasta $15-30 \%$ de los pacientes, como síntoma único o de inicio en la enfermedad leve o como síntoma inicial de pacientes que finalmente presentan insuficiencia respiratoria más grave debido a neumonía atípica. Se ha presentado alteraciones gustativas, como: ageusia, hipogeusia, hipergeusia y disgeusia o parageusia; demostrando que el COVID-19 puede infectar los tejidos orales y causar disfunciones gustativas (Obiefuna \& Donohoe, 2020; Vinayachandran \& Balasubramanian, 2020) Por otro lado, Von Bartheld et al. (2020) hallaron una prevalencia aleatoria de disfunción del gusto del 30,4 \% y que la hipogeusia disminuyó conforme disminuye la gravedad de la enfermedad; además vieron que la etnia influye significativamente en la disfunción del gusto.

Aparte de la pérdida del sentido del gusto, también se considera que el COVID-19 puede ocasionar lesiones secundarias resultantes del deterioro de la salud sistémica o debido a los tratamientos para COVID19 , considerando la posibilidad de infecciones oportunistas y de reacciones adversas de los tratamientos (Amorim Dos Santos et al., 2020).

En este sentido, se han descrito lesiones ulceradas en el paladar duro, lengua y labios, que podrían ser una reacción primaria al SARS-CoV-2, dado que pocos días después de esta lesión el paciente dio posi- 
tivo al COVID-19 considerando que estas lesiones podrían ser un síntoma inicial de esta enfermedad (Soares et al.; Chaux-Bodard et al., 2020).

Amorim Dos santos et al.; señalan que se pueden presentar infecciones fúngicas oportunistas, infección recurrente por el virus del herpes simple oral, ulceraciones orales inespecíficas, disgeusia, ulceraciones y gingivitis, como resultado del sistema inmune deteriorado y la mucosa oral susceptible en pacientes positivos al coronavirus. De forma similar, Sinadinos \& Shelswell (2020) y Carreras-Presas et al. (2020) describieron la presencia de úlceras y ampollas que cursaron con dolor en el paladar, garganta y en la lengua, gingivitis descamativa; pero no determinan si estas son comunes a la infección por SARS-CoV-2 o se dan por la angustia emocional, haciendo hincapié en la importancia de los exámenes intraorales para pacientes afectados por SARS-CoV-2.

También se ha sugerido que la enfermedad periodontal (EP) tiene relación con la gravedad del COVID-19, dado que los pacientes con intubación muestran su salud oral deteriorada produciendo disbiosis de la microbiota oral que podrían desencadenar la EP, gracias a la aparición de periodonto patógenos; por lo tanto, la entrada del virus puede subvertir el sistema inmune y la microbiota oral del huésped, desencadenando la disbiosis que puede permitir la asociación grave de la EP con el COVID-19, por lo que la EP podría ayudar a identificar grupos de riesgo y establecer recomendaciones tempranas (Pitones-Rubio et al., 2020). Así mismo, la mala higiene oral se considera un riesgo para las complicaciones en pacientes predispuestos a biofilms alterados por diabetes, hipertensión o enfermedades cardiovasculares; aumentando el riesgo de infecciones respiratorias y posibles complicaciones bacterianas posvirales, donde las bacterias desempeñan un papel importante para aumentar la posibilidad de complicaciones como neumonía, neumonía aguda, síndrome de dificultad respiratoria, sepsis, shock séptico y muerte (Sampson, 2020). Existiendo varios mecanismos para explicar el potencial de las bacterias orales en la patogénesis de una infección respiratoria: (1) Aspiración de patógenos orales hacia los pulmones. (2) Las enzimas periodontales asociadas a la enfermedad pueden modificar las superficies mucosas para permitir la adhesión y colonización de los patógenos respiratorios. (3) Las enzimas periodontales asociadas a la enfermedad pueden destruir las películas salivales de las bacterias que dificultan su eliminación de las superficies mucosas. (4) EI epitelio respiratorio puede verse alterado por las citocinas periodontales que promueven la infección por patógenos respiratorios (Sampson et al.).

Otro punto, a tomar en cuenta dentro de la cavidad oral, es que la infección grave por COVID-19, junto con las medidas terapéuticas y farmacológicas asociadas, podrían agravar las afecciones orales con etiología de mecanismos inmunes y a la vez, puede interferir con la dinámica del equilibrio de la microbiota que conduzca a varias infecciones micóticas oportunistas, xerostomía relacionada con una disminución del flujo salival, ulceraciones y gingivitis; por lo que se hace necesario monitorear de cerca la salud oral de los pacientes que sean dados de alta (Dziedzic \& Wojtyczka, 2020).

Un efecto secundario que se manifiesta en la mucosa oral por el uso prolongado de los fármacos usados como el difosfato de cloroquina, es la hiperpigmentación del paladar duro, que es indolora y se ve como una mancha de color negro azulado mal delimitada (de Melo et al., 2012; Godinho et al., 2020).

Por último, también se debe considerar a los cambios en el entorno de la vida social y el aumento de la presión laboral ocasionada por el aislamiento social que se usa para combatir el COVID-19, que pueden causar depresión del sistema inmune, produciendo un ataque agudo o la recurrencia de alguna enfermedad crónica de la mucosa oral, como grandes áreas de erosión y ulceración, dificultad de cicatrización, dolor severo, e incluso enfermedades alérgicas, enfermedades infecciosas, liquen plano oral erosivo, úlceras aftosas herpetiformes, pénfigo, etc. (Guo et al., 2020).

\section{CONCLUSIÓN}

La salud oral debe ser parte integral para el manejo del COVID-19 por la presencia de repercusiones que se dan como efecto directo del virus SARS-CoV-2, a consecuencia de los tratamientos a que se someten los pacientes o por descuido de la higiene oral cuando el paciente se encuentra postrado. Por lo que, se hace necesario que los odontólogos estén preparados para realizar un adecuado examen clínico antes de cualquier tratamiento para detectar si hay lesiones en la mucosa oral que pueden ser consideradas como los primeros signos de la infección con SARS-CoV-2; y así, ante cualquier paciente sospechoso solicitar los exámenes auxiliares necesarios para disminuir la posibilidad de contaminación. 
FALCÓN-GUERRERO, B. E. \& FALCÓN-PASAPERA, G. S. Repercussion on the oral cavity caused by COVID-19 infection. Int. J. Odontostomat., 15(1):23-26, 2021.

ABSTRACT: Faced with this situation of the COVID19 pandemic, dentists play an important role in detecting and supporting early diagnosis, since effects that this infection causes in the oral cavity and that are related to the onset are being reported. of this infectious process. These effects can be related to the presence of the SARS-CoV-2 virus or to the forms of treatments that are given to the patient, which are detrimental to the oral health of patients. The objective of this review is to inform about the repercussions that can occur in the oral cavity due to infection with COVID-19.

Keywords: COVID-19, Dental Health Services, SARS-CoV-2; infection control, coronavirus, saliva, dentistry.

\section{REFERENCIAS BIBLIOGRÁFICAS}

Amorim Dos Santos, J.; Normando, A. G. C.; Carvalho da Silva, R. L.; Monteiro De Paula, R.; Cembranel, A. C.; Santos-Silva, A. R. \& Silva Guerra, E. N. Oral mucosal lesions in a COVID-19 patient: New signs or secondary manifestations? Int. J. Infect. Dis., 97:3268, 2020 .

Carreras-Presas, C. M.; Amaro Sánchez, J.; López-Sánchez, A. F.; Jané-Salas, E. \& Somacarrera Pérez, M. L. Oral vesiculobullous lesions associated with SARS-CoV-2 infection. Oral Dis., 2020. DOI: https://www.doi.org/10.1111/odi.13382

Chaux-Bodard, A. G.; Deneuve, S. \& Desoutter, A. Oral manifestation of Covid-19 as an inaugural symptom? J. Oral Med. Oral Surg., 26(2):18, 2020

Cornejo-Ovalle, M. \& Espinoza-Santander, I. COVID-19 and oral manifestations. Int. J. Odontostomat., 14(4):538-9, 2020.

de Melo Filho, M. R.; Dias da Silva, C. A.; da Rocha Dourado, M.; de Oliveira Pires, M. B.; Pêgo, S. P. B. \& de Freitas, E. M. Palate Hyperpigmentation caused by prolonged use of the anti-malarial chloroquine. Head Neck Pathol., 6(1):48-50, 2012.

Dziedzic, A. \& Wojtyczka, R. The impact of coronavirus infectious disease 19 (COVID-19) on oral health. Oral Dis., 2020. DOI: https:/ /www.doi.org/10.1111/odi.13359

Dziedzic, A. special care dentistry and COVID-19 outbreak: what lesson should we learn? Dent. J. (Basel), 8(2):46, 2020.

Falcón-Guerrero, B. E. \& Falcón-Pasapera, G. S. Recommendations for Control of Infection with Novel Coronavirus in Dentistry. J. Dent. Oral Disord., 6(2):1129, 2020.

Godinho, G. V.; Paz, A. L. L. M.; de Araújo Gomes, E. P. A.; Garcia, C. L. \& Volpato, L. E. R. Extensive hard palate hyperpigmentation associated with chloroquine use. Br. J. Clin. Pharmacol., 2020. DOI: https://www.doi.org/10.1111/bcp.14313

Guo, Y.; Yuan, C. \& Wei, C. Emergency measures for acute oral mucosa diseases during the outbreak of COVID-19. Oral Dis., 2020. DOI: https://www.doi.org/10.1111/odi.13350

Harikrishnan, P. Gustatory dysfunction as an early symptom in COVID19 screening. J. Craniofac. Surg., 2020. DOI: https://www.doi.org/ 10.1097/SCS. 0000000000006797

Lee, J. M. \& Lee, S. J. Olfactory and gustatory dysfunction in a COVID19 patient with ankylosing spondylitis treated with etanercept: case report. J. Korean Med. Sci., 35(21):e201, 2020.

Liu, L.; Wei, Q.; Alvarez, X.; Wang, H.; Du, Y.; Zhu, H.; Jiang, H.; Zhou, J.; Lam, P.; Zhang, L.; et al. Epithelial cells lining salivary gland ducts are early target cells of severe acute respiratory syndrome coronavirus infection in the upper respiratory tracts of rhesus macaques. J. Virol., 85(8):4025-30, 2011.

Niazkar, H. R.; Zibaee, B.; Nasimi, A. \& Bahri, N. The neurological manifestations of COVID-19: a review article. Neurol. Sci., 2020. DOI: https://www.doi.org/10.1007/s10072-020-04486-3

Obiefuna, S. \& Donohoe, C. Neuroanatomy, Nucleus Gustatory. In: StatPearls. Treasure Island (FL), StatPearls Publishing; 2020. Disponible en: https://www.ncbi.nlm.nih.gov/books/NBK554522/

Peng, X.; Xu, X.; Li, Y.; Cheng, L.; Zhou, X. \& Ren, B. Transmission routes of 2019-nCoV and controls in dental practice. Int. J. Oral Sci., 12(1):9, 2020.

Qi, F.; Qian, S.; Zhang, S. \& Zhang, Z. Single cell RNA sequencing of 13 human tissues identify cell types and receptors of human coronaviruses. Biochem. Biophys. Res. Commun., 526(1):135-40, 2020.

Pitones-Rubio, V.; Chávez-Cortez, E. G.; Hurtado-Camarena, A.; González-Rascón, A. \& Serafín-Higuera, N. Is periodontal disease a risk factor for severe COVID-19 illness?. Med Hypotheses, 144:109969, 2020

Sabino-Silva, R.; Jardim, A. C. G. \& Siqueira, W. L. Coronavirus COVID19 impacts to dentistry and potential salivary diagnosis. Clin. Oral Investig., 24(4):1619-21, 2020

Sampson, V. Oral hygiene risk factor. Br. Dent. J., 228(8):569, 2020a.

Sampson, V.; Kamona, N. \& Sampson, A. Could there be a link between oral hygiene and the severity of SARS-CoV-2 infections? Br. Dent. J., 228(12):971-5, 2020b.

Sinadinos, A. \& Shelswell, J. Oral ulceration and blistering in patients with COVID-19. Evid. Based Dent. 21(2):49, 2020.

Soares, C. D.; de Carvalho, R. A.; de Carvalho, K. A.; de Carvalho, M. G. F. \& de Almeida, O. P. Letter to Editor: Oral lesions in a patient with Covid-19. Med. Oral Patol. Oral Cir. Bucal, 25(4):e563-4, 2020.

Srinivasan, M.; Zunt, S. L. \& Goldblatt, L. I. Oral epithelial expression of angiotensin converting enzyme-2: Implications for COVID-19 diagnosis and prognosis. bioRxiv, 2020. DOI: https://www.doi.org/ 10.1101/2020.06.22.165035

To, K. K. W.; Tsang, O. T. Y.; Yip, C. C. Y.; Chan, K. H.; Wu, T. C.; Chan, J. M. C.; Leung, W. S.; Chik, T. S. H.; Choi, C. Y. C.; Kandamby, D. $\mathrm{H} . ;$ et al. Consistent detection of 2019 novel coronavirus in saliva. Clin. Infect. Dis., 71(15):841-3, 2020

Vinayachandran, D. \& Balasubramanian, S. Is gustatory impairment the first report of an oral manifestation in COVID-19?. Oral Dis., 2020. DOI: https://www.doi.org/10.1111/odi.13371

von Bartheld, C. S.; Hagen, M. M. \& Butowt, R. Prevalence of Chemosensory Dysfunction in COVID-19 Patients: A Systematic Review and Meta-analysis Reveals Significant Ethnic Differences. medRxiv. 2020. DOI: https://www.doi.org/10.1101/ 2020.06.15.20132134

Xu, H.; Zhong, L.; Deng, J.; Peng, J.; Dan, H.; Zeng, X.; Li, T. \& Chen, Q. High expression of ACE2 receptor of 2019-nCoV on the epithelial cells of oral mucosa. Int. J. Oral Sci., 12:8, 2020a.

Xu, R.; Cui, B.; Duan, X.; Zhang, P.; Zhou, X. \& Yuan, Q. Saliva: potential diagnostic value and transmission of 2019-nCoV. Int. J. Oral Sci., 12:11, 2020b.

Dirección para correspondencia:

Britto Ebert Falcón-Guerrero

Av. Tarapacá \# 350

Tacna

PERÚ

Email: artdent2000@hotmail.com 